\title{
The QCD equation of state for two flavours at non-zero chemical potential
}

S. Ejiri ${ }^{\mathrm{a} *}$, C.R. Allton ${ }^{\mathrm{b}}$, M. Döring ${ }^{\mathrm{cd}}$, S.J. Hands ${ }^{\mathrm{b}}$, O. Kaczmarek ${ }^{\mathrm{c}}$, F. Karsch ${ }^{\mathrm{cd}}$, E. Laermann ${ }^{\mathrm{c}}$ and K. Redlich ${ }^{\mathrm{ef}}$.

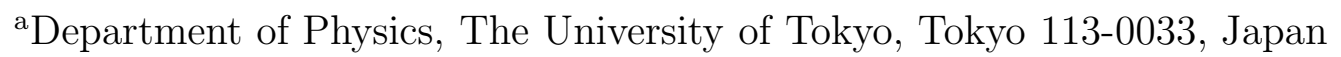

${ }^{\mathrm{b}}$ Department of Physics, University of Wales Swansea, Swansea SA2 8PP, UK

${ }^{\mathrm{c} F a k u l t a ̈ t ~ f u ̈ r ~ P h y s i k, ~ U n i v e r s i t a ̈ t ~ B i e l e f e l d, ~ D-33615 ~ B i e l e f e l d, ~ G e r m a n y ~}$

${ }^{d}$ Physics Department, Brookhaven National Laboratory, Upton, NY 11973, USA

e Institute of Theoretical Physics, University of Wroclaw, PL-50204 Wroclaw, Poland

${ }^{\text {f}}$ Physics Department, Theory Division, CERN, CH-1211 Geneva 23, Switzerland

We present results of a simulation of 2 flavour QCD on a $16^{3} \times 4$ lattice using p4improved staggered fermions with bare quark mass $m / T=0.4$. Derivatives of the thermodynamic grand canonical partition function $Z\left(V, T, \mu_{u}, \mu_{d}\right)$ with respect to chemical potentials $\mu_{u, d}$ for different quark flavours are calculated up to sixth order, enabling estimates of the pressure and the quark number density as well as the chiral condensate and various susceptibilities as functions of $\mu_{u, d}$ via Taylor series expansion. Results are compared to high temperature perturbation theory as well as a hadron resonance gas model. We also analyze baryon as well as isospin fluctuations and discuss the relation to the chiral critical point in the QCD phase diagram. We moreover discuss the dependence of the heavy quark free energy on the chemical potential.

\section{Introduction}

It is important to study QCD at high temperature and high density by numerical simulations of lattice QCD. In particular, studies of the equation of state (EoS) can provide basic input for the analysis of the experimental signatures for QGP formation, e.g. the EoS will control the properties of any hydrodynamic expansion. We extend studies of the EoS to non-zero baryon number density.

Simulation at non-zero baryon density is known to be difficult; however recent studies have found that a Taylor expansion with respect to chemical potential $\mu_{u, d}$ is an efficient technique to investigate the low density regime, interesting for heavy-ion collisions [1], 2]. In the calculation of the Taylor expansion coefficients, i.e. calculation of the derivatives at $\mu_{u, d}=0$, the technical difficulty for non-zero $\mu_{u, d}$ does not arise and a quantitative

${ }^{*}$ Speaker. SE is supported by Grants-in-Aid of the Japanese MEXT No. 15540254. KR is supported by KBN under grant 2P03 (06925). FK is partly supported by a contract DE-AC02-98CH1-886 with the U.S. DOE. MD is supported by DFG grant GRK- 881 . 

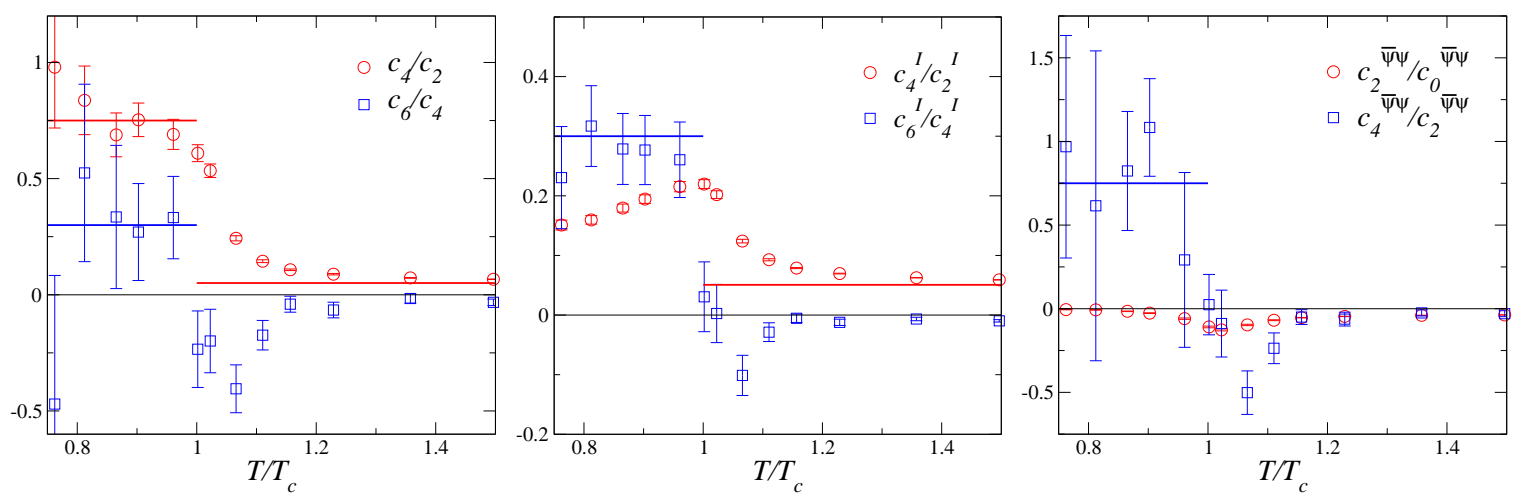

Figure 1. The ratios of Taylor expansion coefficients for pressure (quark number susceptibility), isospin susceptibility and chiral condensate.

study becomes possible within the error by truncation of higher order terms. Since thermodynamic quantities can be defined by derivatives of the partition function, e.g. chiral condensate $\langle\bar{\psi} \psi\rangle=(T / V)(\partial \ln Z / \partial m)$, quark number density $n_{q}=(T / V)\left(\partial \ln Z / \partial \mu_{q}\right)$, quark number susceptibility $\chi_{q}=(T / V)\left(\partial^{2} \ln Z / \partial \mu_{q}^{2}\right)$, and isospin susceptibility $\chi_{I}=$ $(T / V)\left(\partial^{2} \ln Z / \partial \mu_{I}^{2}\right)$, where $\mu_{q}=\left(\mu_{u}+\mu_{d}\right) / 2$ and $\mu_{I}=\left(\mu_{u}-\mu_{d}\right) / 2$, the calculation of first derivatives yields basic QCD thermodynamics observables, and the calculation of higher derivatives is a natural extension.

In this study, we evaluate the Taylor expansion coefficients of thermodynamic quantities and compare these results to the predictions from the perturbation theory in the high temperature limit and from the hadron resonance gas model in the low temperature hadron phase. Also fluctuations of quark number, isospin number and electric charge are discussed near the transition temperature $T_{c}$. They are estimated by $\chi_{q}, \chi_{I}$ and charge susceptibility $\chi_{C}=\chi_{q} / 36+\chi_{I} / 4$, and are related to the critical endpoint expected at non-zero $\mu_{q}$. Moreover we study the Taylor expansion coefficients of chiral condensate and heavy quark free energy. The details of simulations are given in [2].

\section{Quark gluon gas and hadron resonance gas}

We define the expansion coefficients by $p / T^{4}=(\ln Z) /\left(V T^{3}\right) \equiv \sum_{n=0}^{\infty} c_{n}\left(\mu_{q} / T\right)^{n}$, $\chi_{q}\left[\chi_{I}\right] / T^{2} \equiv \sum_{n=2}^{\infty} n(n-1) c_{n}\left[c_{n}^{I}\right]\left(\mu_{q} / T\right)^{n-2}$ and $\langle\bar{\psi} \psi\rangle / T^{3} \equiv \sum_{n=0}^{\infty} c_{n}^{\bar{\psi} \psi}\left(\mu_{q} / T\right)^{n}$ for $\mu_{I}=0$. We expect the equation of state to approach that of a free quark-gluon gas (StefanBoltzmann (SB) gas) in the high temperature limit. The coefficients in the SB limit for 2 flavour QCD with $\mu_{I}=0$ are well known as $c_{2}=c_{2}^{I}=1, c_{4}=c_{4}^{I}=1 /\left(2 \pi^{2}\right)$ and $c_{n}=c_{n}^{I}=0$ for $n \geq 6$. Moreover the sign of $c_{6}$ is negative starting at $O\left(g^{3}\right)$ in perturbation theory. On the other hand, in the low temperature phase QCD is well described by a hadron resonance gas model in which the pressure is obtained by summing over the contributions from all resonance states of hadrons. In this model, the contribution to $p / T^{4}$ from each individual hadron which has baryon number $B_{i}$ is in proportion to $\exp \left(3 B_{i} \mu_{q} / T\right)$, hence the pressure can be written as $p / T^{4}=G(T)+F(T) \cosh \left(3 \mu_{q} / T\right)$, where $G(T)$ and $F(T)$ are the mesonic and baryonic components of $p / T^{4}$ at $\mu_{q}=0$. Similarly, we obtain $\chi_{q} / T^{2}=9 F(T) \cosh \left(3 \mu_{q} / T\right), \chi_{I} / T^{2}=G^{I}(T)+F^{I}(T) \cosh \left(3 \mu_{q} / T\right)$ 

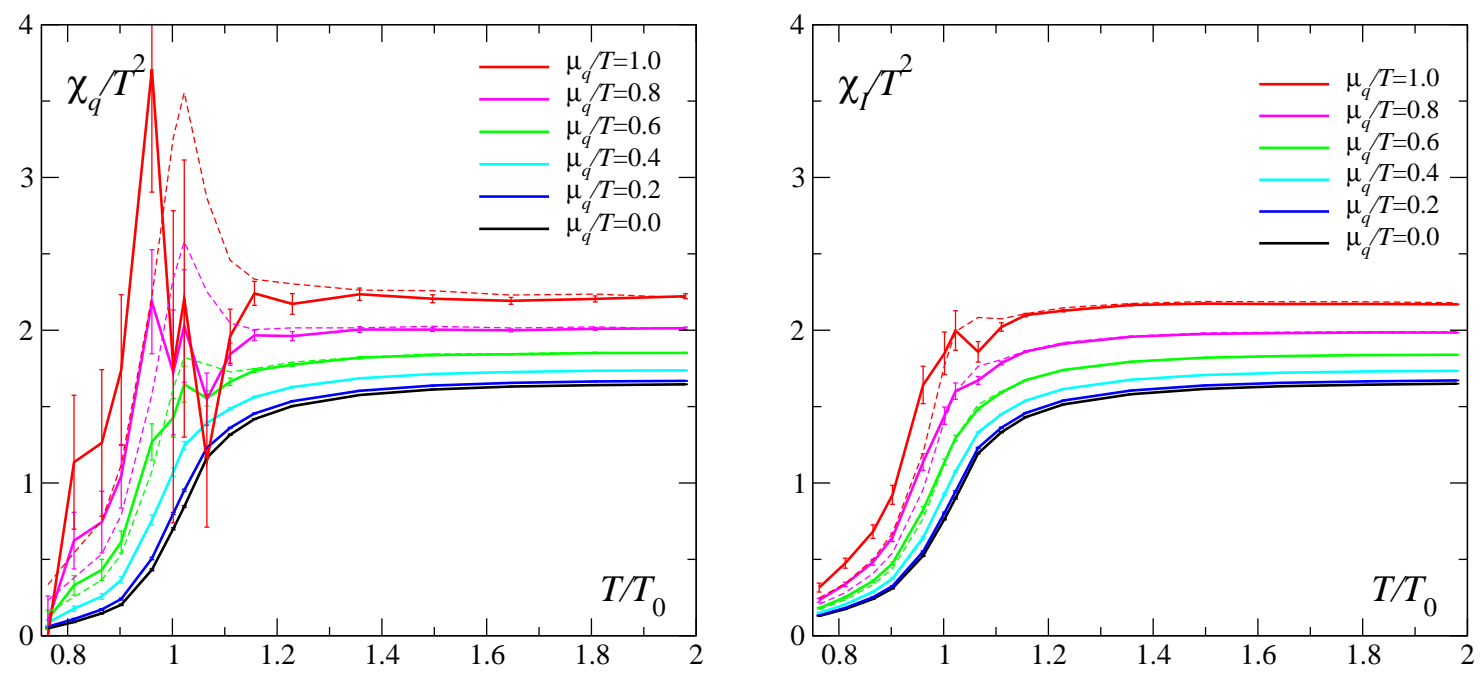

Figure 2. The quark number susceptibility $\chi_{q} / T^{2}$ (left) and isospin susceptibility $\chi_{I} / T^{2}$ (right) for various $\mu_{q} / T$. $T_{0}$ is $T_{c}$ at $\mu_{q}=0$.

and $\langle\bar{\psi} \psi\rangle / T^{3}=G^{\bar{\psi} \psi}(T)+F^{\bar{\psi} \psi}(T) \cosh \left(3 \mu_{q} / T\right)$. Here, the mesonic component for $\chi_{q}$ is zero because mesons have baryon number zero. Therefore, $c_{4} / c_{2}=3 / 4, c_{6} / c_{4}=3 / 10$, $c_{6}^{I} / c_{4}^{I}=3 / 10$ and $c_{4}^{\bar{\psi} \psi} / c_{2}^{\bar{\psi} \psi}=3 / 4$ in the region where the hadron resonance gas provides a good approximation.

We investigate these coefficients. The results for $c_{n+2} / c_{n}, c_{n+2}^{I} / c_{n}^{I}$ and $c_{n+2}^{\bar{\psi} \psi} / c_{n}^{\bar{\psi} \psi}$ are shown in Fig. 1. We find that these results are consistent with the prediction from the hadron resonance gas model for $T / T_{c} \leq 0.96$ and approach the $\mathrm{SB}$ values, i.e. $c_{4} / c_{2}=$ $c_{4}^{I} / c_{2}^{I}=1 / 2 \pi^{2}, c_{6} / c_{4}=c_{6}^{I} / c_{4}^{I}=0$, in the high temperature limit. Also $c_{6}$ is negative at high temperature as expected in the perturbation theory. These results suggest that the models of free quark-gluon gas and hadron gas seem to explain the behaviour of thermodynamic quantities well except in the narrow regime near $T_{c}$.

\section{Susceptibilities at non-zero $\mu_{q}$}

Next, we calculate quark number and isospin susceptibilities in a range of $0 \leq \mu_{q} / T \leq 1$. The data connected by solid lines in Fig. 2 are obtained by $\chi_{q} / T^{2}=2 c_{2}+12 c_{4}\left(\mu_{q} / T\right)^{2}+$ $30 c_{6}\left(\mu_{q} / T\right)^{4}$ and the corresponding equation for $\chi_{I}$. Dashed lines are the results from $O\left(\mu_{q}^{2}\right)$ expansion. Since the statistical error of $c_{6}$ is still large near $T_{c}$, the location and height of the peak are less accurately determined, and also the error from the truncation of higher order terms of $\mu_{q} / T$ seems to be visible for large $\mu_{q} / T$. However, as seen in Fig. 1. $c_{6}$ changes its sign at $T_{c}$. This means the peak position of $\chi_{q}$ moves left, which is corresponding to the change of $T_{c}$ as a function of $\mu_{q} . T_{c}\left(\mu_{q} / T=1\right) / T_{c}\left(\mu_{q} / T=0\right)$ in [ 3] is about 0.93. Moreover, $\chi_{q}$ increases with higher orders of the expansion for $T \leq T_{c}$ which confirms the existence of a peak. This suggests the presence of a critical endpoint in the $\left(T, \mu_{q}\right)$ plane. On the other hand, $\chi_{I}$ in Fig. 2 does not show any singular behaviour. This is consistent with the sigma model prediction that only isosinglet degrees of freedom become massless at the critical endpoint. 


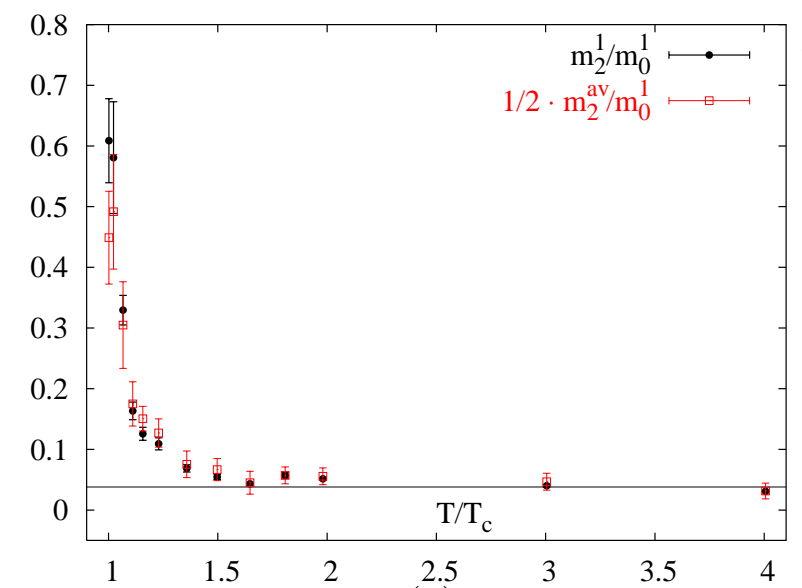

(a)

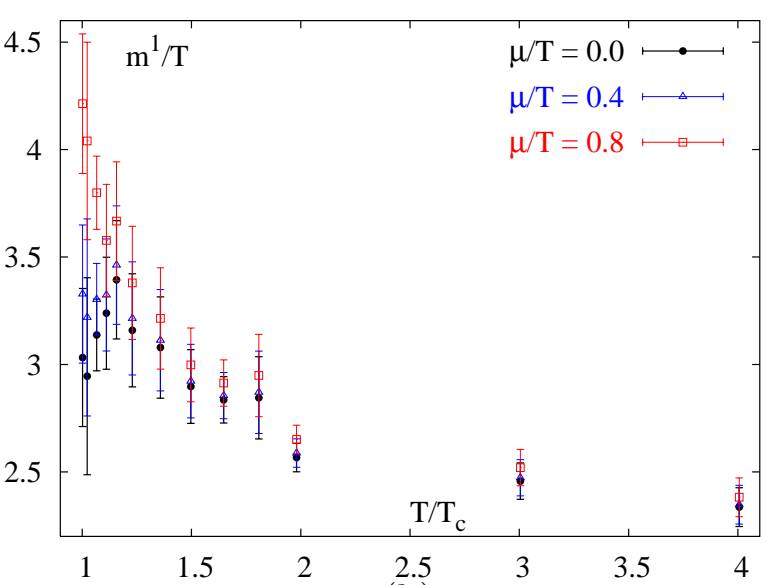

(b)

Figure 3. (a) The ratios $m_{2}^{1} / m_{0}^{1}$ and $m_{2}^{\text {av }} / m_{0}^{1}$ as functions of $T / T_{c}$ and (b) the singlet screening mass at non-zero $\mu_{q}$.

\section{Screening mass at non-zero $\mu_{q}$}

Finally we want to discuss the free energy of a static quark anti-quark pair at finite temperature and density. We extract the singlet free energy $F_{Q \bar{Q}}^{1}$ and colour averaged free energy $F_{Q \bar{Q}}^{\mathrm{av}}$ by the Polyakov loop correlation functions. For $T>T_{c}$, the free energy is expected to be exponentially screened at large distances, $\Delta F_{Q \bar{Q}}^{\mathrm{av}, 1}\left(r, T, \mu_{q}\right) \equiv F_{Q \bar{Q}}^{\mathrm{av}, 1}\left(\infty, T, \mu_{q}\right)-$ $F_{Q \bar{Q}}^{\mathrm{av}, 1}\left(r, T, \mu_{q}\right) \sim e^{-m^{\mathrm{av}, 1}\left(T, \mu_{q}\right) r}$. We calculate the Taylor expansion coefficients of the Debye screening mass $m^{\mathrm{av}, 1}$ in terms of $\mu_{q} / T$ at $\mu_{q}=0, m^{\mathrm{av}, 1} \equiv \sum_{n=0}^{\infty} m_{n}^{\mathrm{av}, 1}\left(\mu_{q} / T\right)^{n}$. We plot the data of $m_{2}^{1} / m_{0}^{1}$ and $m_{2}^{\text {av }} / m_{0}^{1}$ in Fig. 3(a). We find $m_{2}^{1}=(1 / 2) m_{2}^{\text {av }}$. This is expected from perturbation theory, which suggests that the leading order contribution to the colour singlet free energy is given by one gluon exchange while the colour averaged free energy is dominated by two gluon exchange. Moreover the perturbative Debye screening mass $m_{D}$ is given by $m_{D}\left(T, \mu_{q}\right)=m_{D, 0}(T) \sqrt{1+3 N_{f} /\left[\left(2 N_{c}+N_{f}\right) \pi^{2}\right]\left(\mu_{q} / T\right)^{2}}$, with $m_{D, 0}(T)=g(T) T \sqrt{\left(2 N_{c}+N_{f}\right) / 6}$. Here $N_{c}$ and $N_{f}$ are the number of colour and flavour respectively. The solid line in Fig. 3 (a) is the perturbative prediction for $m_{2}^{1} / m_{0}^{1}$. The ratio $m_{2}^{1} / m_{0}^{1}$ is found to be consistent with perturbation theory for $T \geq 2 T_{c}$. In Fig. 3 (b) we show the $\mu_{q}$-dependence of the singlet screening mass $m^{1}\left(\mu_{q}, T\right) / T$ for a small values of $\mu_{q} / T$, where only contributions from $m_{0}^{1}$ and $m_{2}^{1}$ are included. Further details of this study are given in [4].

\section{REFERENCES}

1. C.R. Allton, S. Ejiri, S.J. Hands, O. Kaczmarek, F. Karsch, E. Laermann and C. Schmidt, Phys. Rev. D68 (2003) 014507.

2. C.R. Allton, M. Döring, S. Ejiri, S.J. Hands, O. Kaczmarek, F. Karsch, E. Laermann and K. Redlich, Phys. Rev. D71 (2005) 054508.

3. C.R. Allton, S. Ejiri, S.J. Hands, O. Kaczmarek, F. Karsch, E. Laermann, Ch. Schmidt and L. Scorzato, Phys. Rev. D66 (2002) 074507.

4. M. Döring, S. Ejiri, O. Kaczmarek, F. Karsch and E. Laermann, hep-lat/0509001. 